\title{
Population-Based Tuberculosis Disease Prevalence Survey in Ghana: The Role and Lessons Learnt from the Laboratory
}

\author{
Kennedy Kwasi Addo1*, Samuel Ofori Addo1, Christian Bonsu', Ezekiel Mensah¹, Sarah Edusei1, \\ Prosper Dedzo', Michael Amo Omari², Samuel Kudzawu², Honesty Ganu², \\ Samuel Kumah Atiadevie'2, Frank Adae Bonsu ${ }^{3}$
}

${ }^{1}$ Department of Bacteriology, Noguchi Memorial Institute for Medical Research, College of Health Sciences, University of Ghana, Accra, Ghana

${ }^{2}$ Chest Clinic Laboratory, Korle-Bu Teaching Hospital, Accra, Ghana

${ }^{3}$ National Tuberculosis Control Programme, Accra, Ghana

Email: *kaddo@noguchi.ug.edu.gh

How to cite this paper: Addo, K.K., Addo, S.O., Bonsu, C., Mensah, E., Edusei, S., Dedzo, P., Omari, M.A., Kudzawu, S., Ganu, H., Atiadevie, S.K. and Bonsu, F.A. (2019) Population-Based Tuberculosis Disease Prevalence Survey in Ghana: The Role and Lessons Learnt from the Laboratory. Journal of Tuberculosis Research, 7, 95-108. https://doi.org/10.4236/jtr.2019.72009

Received: May 22, 2019

Accepted: June 27, 2019

Published: June 30, 2019

Copyright $\odot 2019$ by author(s) and Scientific Research Publishing Inc. This work is licensed under the Creative Commons Attribution International License (CC BY 4.0).

http://creativecommons.org/licenses/by/4.0/ (c) (i) Open Access

\begin{abstract}
Background: Bacteriologically-confirmed tuberculosis (TB) cases used in calculating TB prevalence in a country are obtained through laboratory examination of sputum specimens. Objective: This article describes laboratory processing of specimens, results overview, conclusions and key lessons learnt from the perspective of laboratory personnel involved in the conduct of TB disease prevalence survey in Ghana in 2013. Methods: Symptoms screening and Chest X-ray suggestive of TB were used to select participants who produced sputum to confirm TB cases using microscopy, culture and Xpert ${ }^{\circledR}$ MTB/RIF assay (GeneXpert). Results: A total of 15,935 single and paired sputum specimens were received from eligible participants. About half of Ziehl-Nielsen (129/263) and Auramine O (122/246) stained smear positives were scanty positive. Culture positivity rate for Mycobacterium tuberculosis complex was 266/14,994 (1.7\%) and 100/15,179 (0.7\%) in Mycobacterial Growth Indicator Tube (MGIT) and Lowenstein-Jensen (LJ) media respectively; while non-tuberculous mycobacterium was 294/14,994 (1.96\%) and 167/15,179 (1.1\%). Total contamination rates in MGIT (5.4\%) were higher than in LJ (1.7\%). Prevalence of smear positive TB and bacteriologically confirmed TB among adult population ( $\geq 15$ years) was estimated at 111 (95\% CI: 76 - 145) and 356 (95\% CI: 288 - 425) per 100,000 population respectively. Conclusions and Lessons Learnt: Direct supervision of specimen collection by well-trained laboratory personnel, timely transportation of specimens from field to laboratory, prompt specimen processing and use of electronic data management systems are essential for a reliable TB disease prevalence
\end{abstract}


survey data. More importantly, strengthening human and logistical capacity of the laboratory must be of utmost priority.

\section{Keywords}

Prevalence Survey, Tuberculosis Laboratory, Lessons Learnt, Ghana

\section{Introduction}

Despite being declared a global health emergency in 1993 by the World Health Organization (WHO), tuberculosis (TB) remains a disease of public health importance globally infecting about one third of the world's population with about two million deaths occurring annually [1]. In Ghana, an estimated 14,668 (Pulmonary, bacteriologically confirmed-7682; Pulmonary, clinically diagnosed5364; Extra-pulmonary-1181; Relapsed-441) cases were reported in 2014 [2]. While trend analysis of programmatic data provides useful information to indirectly assess progress, there are limitations. Firstly, we are unable to entirely rely on the routine surveillance system owing to its coverage, completeness and accuracy. Secondly, the current WHO estimates may be unrealistic; since the last well conducted prevalence survey based on TB disease in Ghana was in 1957. In the year 2000, TB burden measurement was done, but was based on TB infection, which is less reliable and not very accurate. As of 2012, TB case detection rate in Ghana was very low at 33\% with estimated prevalence of more than 70 per 100,000 population [3]. With these aforementioned reasons, it was imperative for a prevalence survey to be conducted. Therefore, in 2013, a population-based TB disease prevalence survey was conducted with a general objective to obtain a direct measurement of the absolute burden of disease caused by TB in Ghana. A combination of symptom screening using standardized questionnaire and chest X-ray was used in order to selectively target the survey participants who provided sputum for bacteriological tests. We report here the general overview of the prevalence survey process with emphasis on the laboratory activities including sample collection and processing, data obtained, analysis and interpretation, as well as lessons learnt.

\section{Methods}

\subsection{Study Design}

The study was cross sectional and population based carried out on a random sample of the general adult population (15 years and above) in Ghana, in which the number of people with TB disease was measured. There were 98 survey clusters in two strata (urban-53, rural-45).

\subsection{Sample Size}

The overall estimated sample size was 63,905 with an estimated cluster size of 
650 people.

The parameters used to calculate the sample size and underlying assumptions are described as follows:

Smear-positive prevalence for $\geq 15$ years ( 270 per 100,000 population);

Precision (0.2);

Design effect (1.44);

$k^{2}(0.5)$; where $k$ is the coefficient of between-cluster variation

Response rate (85\%).

\subsection{Selection of Eligible Participants}

All inhabitants including visitors in a cluster were enumerated by households prior to the survey. After enumeration, the survey team selected eligible participants from each household based on study inclusion criteria and provided invitation cards to participate in the survey.

\subsubsection{Inclusion Criteria}

All inhabitants of selected clusters who were 15 years old and above; residents and visitors who have lived in the household for the most of the last two (2) weeks preceding survey census (which means at least 7 days or more in the last 14 days); permanent residents who have lived in the household few days (less than 7 days) in the last 2 weeks before the census (i.e. residents who were away for some time, but that just came back home a couple of days before the census).

\subsubsection{Exclusion Criteria}

Eligible residents who are incapable of consenting (mentally incapacitated, people between 15 - 17 years who cannot secure consent etc.); visiting residents who have lived in the enumeration area for less than 7 days in the last 2 weeks prior to census day; permanent resident who have not lived in the household in the last 2 weeks before the census (i.e. people who travelled since more than 2 weeks before the census); hotel guests; prisoners; students in hostels; residents of student dormitories in boarding school premises; residents of seminaries, sisters convents and monastries; diplomatic compounds; military facilities; street dwellers (with no fixed address).

\subsection{Screening Strategy}

Symptom screening and/or chest X-ray and/or chest X-ray exemption were used to identify individuals eligible to produce sputum for bacteriological examinations as recommended by WHO [4]. The overall algorithm for screening eligible participants and specimen processing is shown in Figure 1.

\subsection{Laboratory Technical Staff and Sample Processing Sites}

Two groups of well-trained laboratory technicians and biomedical scientists were fully engaged for the study. The first group consisted of one technician attached to each of the four field survey teams to supervise the collection of sputum 


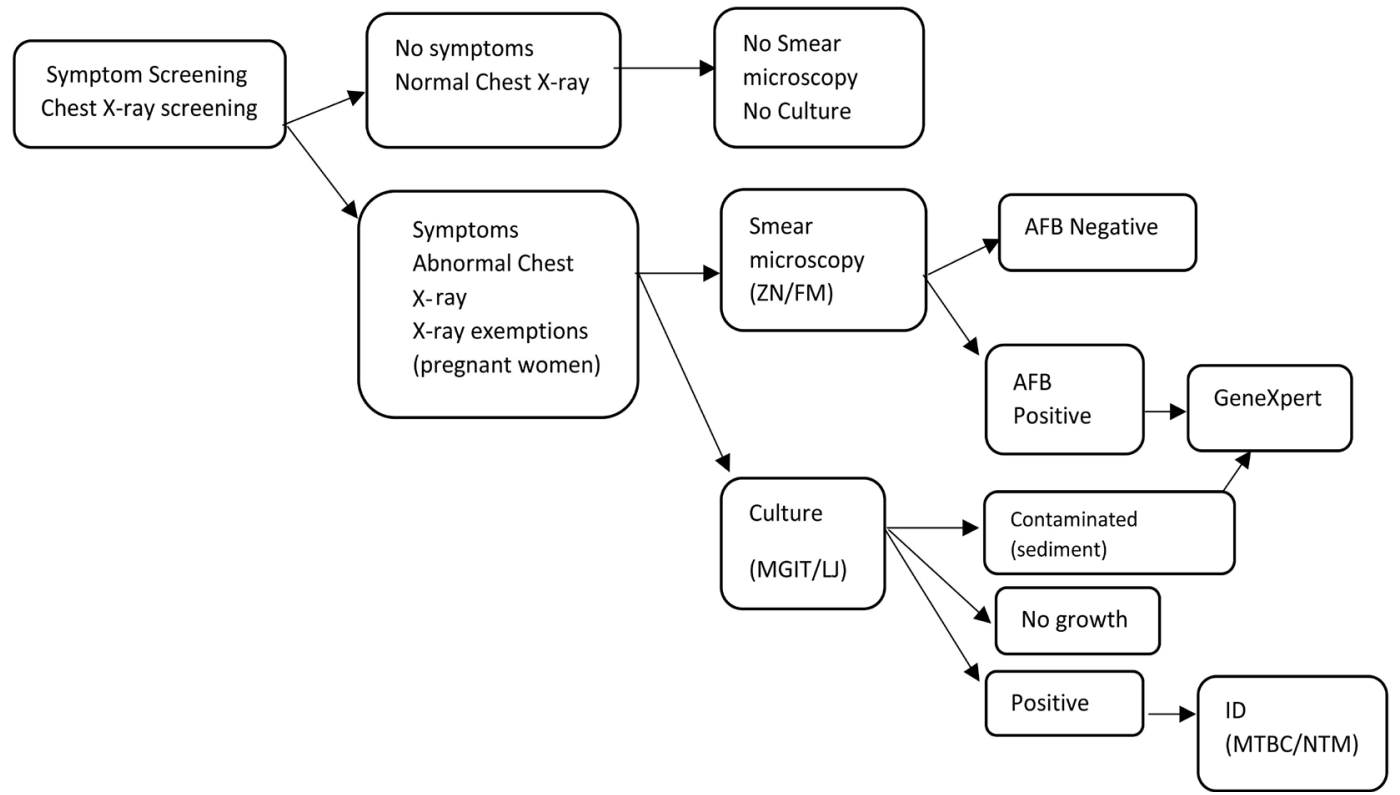

AFB: Acid Fast Bacilli; FM: Fluorescence Microscopy; GeneXpert: Xpert ${ }^{\circledR}$ MTB/RIF assay; ID: Identification; LJ: Lowenstein-Jensen; MGIT: Mycobacterial Growth Indicator Tube; MTBC: Mycobacterium tuberculosis complex; NTM: Non-tuberculous mycobacteria; ZN: Ziehl-Nielsen.

Figure 1. Algorithm for participant screening and specimen processing.

from eligible participants. The other group was based at two laboratories-a biosafety level 3 (BSL3) TB laboratory of Noguchi Memorial Institute for Medical Research (NMIMR) and the Chest Clinic laboratory of the Korle-Bu Teaching Hospital (KBTH) to examine the sputum specimens by microscopy, culture and Xpert ${ }^{\circledR}$ MTB/RIF assay (GeneXpert) (Cepheid, Sunnyvale, CA, USA). NMIMR is a biomedical research facility at the University of Ghana that conducts research, mainly into infectious diseases. The Chest Clinic laboratory is a specialist diagnostic centre at KBTH that receives and process clinical specimens from suspected cases of TB and other chest diseases. These laboratories serve as national reference laboratories for TB in Ghana and subscribe to proficiency testing scheme for TB microscopy and culture from the World Health Organization/National Institute for Communicable Diseases (WHO/NICD) in South Africa and WHO Supranational TB reference Laboratory in Borstel, Germany respectively.

\subsection{Bacteriological Investigations}

\subsubsection{Sputum Sample Collection and Processing}

Two sputum specimen (spot and morning) were collected from each eligible participant according to symptoms screening or abnormal chest radiograph, and from all pregnant women who were exempted from chest X-ray. All the sputum specimens collected from participants in a particular survey cluster were transported in cold chain within $24-48$ hours to NMIMR and KBTH Chest Clinic laboratories for concentrated Ziehl Nielsen (ZN) and Auramine O (AO) stained smear microscopy, Mycobacterial Growth Indicator Tube (MGIT) and Lowenstein-Jensen (LJ) culture as well as GeneXpert. All specimens were processed 
within one week from the date of collection in a Biosafety cabinet class II.

\subsubsection{Sputum Digestion and Decontamination}

Sputum specimen were first processed using Mycoprep ${ }^{\text {Tw }}$ (BD Diagnostic System, Sparks, MD, USA) which is a commercially available Sodium hydroxide- $\mathrm{N}$-acetyl L-cysteine (NaOH-NALC) formulation for sputum digestion and decontamination. This initial process was done according to manufacturer's instructions. In brief, equal volumes of specimen $(3-5 \mathrm{ml})$ and $\mathrm{NaOH}$-NALC solution were mixed together and the preparation allowed to stand for 15 minutes at room temperature $\left(25^{\circ} \mathrm{C}\right)$. Next, about $40-45 \mathrm{ml}$ of phosphate buffer saline (PBS) solution with $\mathrm{pH} 6.8$ was added to the decontaminated specimen and allowed to stand for 20 minutes to neutralize the reaction. Further, the whole preparation was centrifuged at $3000 \times \mathrm{g}$ for 15 minutes to concentrate the specimen and also wash the $\mathrm{NaOH}$ solution. Then the supernatant was discarded to obtain sediment. Finally, a small volume ( $2 \mathrm{ml}$ ) of PBS ( $\mathrm{pH} 6.8$ ) was added to the sediment to make the inoculum for smears and cultures.

\subsubsection{Smear Preparation and Microscopy}

Two smears were prepared from each inoculum and stained for microscopy using $\mathrm{ZN}$ and Auramine $\mathrm{O}$ staining methods. The smears were examined after staining for the presence or absence of acid fast bacilli (AFB). The ZN stained smear was examined using Olympus ${ }^{\mathrm{Tu}}$ light microscope (Olympus Corporation, Shinjuku, Tokyo, Japan) with oil immersion at 1000x and the Auramine O stained smear examined with Primo Star iLED ${ }^{\mathrm{Tw}}$ microscope (Carl Zeiss Microimaging, Oberkochen, Germany), without oil immersion at 400×. Smears were graded using the World Health Organization and the International Union against Tuberculosis and Lung Diseases (WHO/IUALTD) standards. All smear positive specimens and contaminated culture (where sediments were available) were run on GeneXpert for Mycobacterium tuberculosis confirmation as well as rifampicin resistance.

\subsubsection{Culture and Identification of Mycobacterial Species}

Two tubes each of commercially available MGIT medium and LJ slants (BD Diagnostic System, Sparks, MD, USA) were inoculated with $0.5 \mathrm{ml}$ of decontaminated sputum specimen per tube and incubated at $37^{\circ} \mathrm{C}$ (maximum of 8 weeks) and BACTEC MGIT 960 system (6 weeks) respectively. Growth of mycobacteria and other bacteria in the MGIT was indicated by increasing fluorescence. Once a tube flagged positive, smear was prepared, stained by ZN method and examined microscopically for presence or absence of AFBs. In addition, a portion of the positive culture was streaked onto blood agar plates. Growth on the plates indicates contamination. LJ growth was indicated by visible colonies on the slants.

All pure mycobacterial isolates obtained from both MGIT and LJ culture were identified broadly as Mycobacterium tuberculosis complex (MTBC) and Nontuberculosis mycobacterium (NTM) using BD MGIT TBc ID test kit (BD Diag- 
nostic System, Sparks, MD, USA). The test was performed according to manufacturer's instruction. Briefly, each TBC ID test kit was inoculated with $100 \mu \mathrm{l}$ of a positive MGIT culture or in the case of LJ culture, one loopful of colonies suspended in $200 \mu \mathrm{l}$ of extraction buffer (phosphate buffer with $0.05 \%$ Tween 20 and $0.02 \%$ sodium azide) and $100 \mu \mathrm{l}$ of the suspension used in the assay. The results were interpreted $15 \mathrm{~min}$ after application of the sample. A positive result was indicated by the development of two pink bands, one in the control zone (C) and another in the test zone $(\mathrm{T})$.

\subsubsection{Laboratory Case Definitions}

Culture-confirmed TB case: isolation of MTBC from a sputum specimen grown on either LJ, MGIT or both.

Culture confirmed non-TB case: isolation of NTM from a sputum specimen grown on either LJ, MGIT or both.

Sputum smear-positive TB case: acid-fast bacilli (AFB)-positive by $\mathrm{ZN}$ or $\mathrm{Au}$ ramine-O stained sputum smear examination, i.e. at least one AFB in $100 \mathrm{im}$ mersion fields.

\subsubsection{Quality Assurance}

Standard procedures were strictly followed for all laboratory investigations. New batches of all stains used were tested with known positive and negative control slides. Reference TB strains $\left(\mathrm{H}_{37} \mathrm{Rv}\right)$ were inoculated with each new batch of MGIT tubes and LJ slants to assess the medium quality. A senior laboratory technical officer at KBTH Chest Clinic re-checked all positive slides and ten percent of negative slides. An external senior microbiologist from the Kumasi Centre for Collaborative Research (KCCR), Ghana rechecked ten percent of all examined slides available as at the end of the first quarter of the survey period. Laboratory and data analysis experts from WHO visited KBTH and NMIMR TB laboratories on three occasions for monitoring and evaluation.

\subsection{Data Management and Analysis}

All data collected from the field and laboratory were transferred electronically to a central server hosted at the secretariat of the National Tuberculosis Programme (NTP). Data analysis was performed using Stata (v12, Stata Corporation, USA) and SPSS (v20, IBM Corporation, USA).

\subsection{Ethical Consideration}

The study obtained ethical approval from the Institutional Review Board of Noguchi Memorial Institute for Medical Research [NMIMR-IRB CPN: 031/10-11 amend 2012].

\section{Results}

Among 67,757 eligible adults invited, 61,726 (91.1\%) made up of 24,688 males and 37,038 females participated in the study. Majority of the participants, 
$33,122 / 61,626$ (54\%) were urban residents. The major reported occupation of participants was farming (29\%) followed by trading $(24 \%)$. Of the total participants, $48(0.1 \%)$ reported that they were receiving TB treatment at the time of the survey while $331(0.5 \%)$ said they had been treated for TB at least once in their life (Table 1). After symptom screening and/or chest X-ray and/or chest

Table 1. Socio-demographic and clinical characteristics of study participants $(N=61,726)$.

\begin{tabular}{|c|c|c|}
\hline Characteristic & $\mathrm{N}$ & $\%$ \\
\hline \multicolumn{3}{|l|}{ Sex } \\
\hline Male & 24,668 & 40 \\
\hline Female & 37,038 & 60 \\
\hline \multicolumn{3}{|l|}{ Age group (years) } \\
\hline $15-24$ & 17,089 & 27.7 \\
\hline $25-34$ & 13,584 & 22 \\
\hline $35-44$ & 11,057 & 18 \\
\hline $45-54$ & 8689 & 14 \\
\hline $55-64$ & 5442 & 8.8 \\
\hline$\geq 65$ & 5868 & 9.5 \\
\hline \multicolumn{3}{|l|}{ Place of residence } \\
\hline Urban & 33,122 & 53.7 \\
\hline Rural & 28,604 & 46.3 \\
\hline \multicolumn{3}{|l|}{ Occupation } \\
\hline Farmer & 17,760 & 28.8 \\
\hline Trader & 14,831 & 24 \\
\hline Student & 10,448 & 16.9 \\
\hline Artisan & 6450 & 10.5 \\
\hline Civil/Public servant & 4189 & 6.8 \\
\hline Unemployed & 3262 & 5.3 \\
\hline Fisherman & 3216 & 5.2 \\
\hline Pensioner & 1189 & 1.9 \\
\hline Miner & 375 & 0.6 \\
\hline Other ${ }^{a}$ & 6 & 0 \\
\hline \multicolumn{3}{|c|}{ Current anti-TB treatment ${ }^{b}$} \\
\hline Yes & 48 & 0.1 \\
\hline No & 61678 & 99.9 \\
\hline \multicolumn{3}{|c|}{ Previously treated for $\mathrm{TB}^{c}$} \\
\hline Yes & 331 & 0.5 \\
\hline No & 61395 & 99.5 \\
\hline
\end{tabular}

${ }^{a}$ Any other occupation not stated above as used in the study questionnaire. ${ }^{b}$ Number of participants who reported that they were receiving TB treatment at the time of the survey. Number of participants who reported that they had been treated for TB at least once in their life. 
X-ray exemption, a total of 8298 participants were eligible for sputum examination. Out of this number, 8126 (98\%) submitted at least one specimen (either spot, morning or both). About half of the smear positives were of the scanty positive grade (Table 2 ). Of the 126 spot $\mathrm{ZN}$ positive and 137 morning $\mathrm{ZN}$ positive, $36 / 126(28.6 \%)$ and $45 / 137(32.8 \%)$ as well as $48 / 126(38.1 \%)$ and 52/137 (38.0\%) were also positive for MGIT and GeneXpert respectively. Overall, 266/14,994 (1.7\%) and 100/15,179 (0.65\%) specimens were MGIT and LJ culture positive for MTBC respectively. On the other hand, 294/14,994 (1.96\%) and 167/15,179 (1.1\%) NTM were isolated from MGIT and LJ cultures respectively. Contamination rates for specimen per tube were: [MGIT Spot-993/7282 (13.6\%); Morning-1307/7712 (16.9\%)] and LJ [Spot-423/7289 (5.8\%); Morning-654/7720 (8.5\%)]. The rate of total contamination (both spot and morning tubes contaminated) was $417 / 7706$ (5.4\%) and 134/7706 (1.7\%) in MGIT and LJ culture respectively (Table 3 ). A total of 1034 specimens (both single and paired)

Table 2. Acid-fast Bacilli (AFB) detection using Ziehl-Nielsen (ZN) and Auramine O (AO) staining methods.

\begin{tabular}{|c|c|c|c|c|}
\hline \multirow{2}{*}{ Sputum Grade ${ }^{a}$} & \multicolumn{4}{|c|}{ Sputum type/staining method } \\
\hline & Spot ZN & Morning ZN & Spot AO & Morning AO \\
\hline Negative & 8033 & 7591 & 8037 & 7603 \\
\hline Scanty & 63 & 66 & 68 & 63 \\
\hline $1+$ & 24 & 21 & 16 & 17 \\
\hline $2+$ & 20 & 17 & 12 & 10 \\
\hline $3+$ & 19 & 33 & 26 & 34 \\
\hline
\end{tabular}

${ }^{a}$ World Health organization and the International Union against Tuberculosis and Lung Diseases (WHO/IUALTD) standards. Bright field microscopy-ZN stained $(1000 \times, 1$ length $=100$ High Power Field $\{\mathrm{HPF}\})$. Negative $=$ No AFBs/1 length; Scanty = $1-9$ AFB/1 length; $1+=10-99$ AFB/1 length; $2+=1-10$ $\mathrm{AFB} / 1 \mathrm{HPF}$ on average; $3+\geq 10 \mathrm{AFB} / 1 \mathrm{HPF}$ on average. iLED Fluorescence microscopy-AO stained (400×, 1 length $=40$ fields $=200 \mathrm{HPF})$. Negative $=$ No AFBs/1 length; Scanty = $1-19$ AFB/1 length; $1+=20-199$ $\mathrm{AFB} / 1$ length; $2+=5-50 \mathrm{AFB} / 1$ field on average; $3+\geq 50 \mathrm{AFB} / 1$ field on average. AO: Auramine O; $\mathrm{ZN}$ : Ziehl-Nielsen.

Table 3. Growth outcomes from specimen types and culture methods.

\begin{tabular}{ccccc}
\hline & \multicolumn{2}{c}{ MGIT } & LJ \\
\cline { 2 - 5 } & Spot $^{a}, \mathrm{~N}(\%)$ & Morning $^{b}, \mathrm{~N}(\%)$ & Spot $^{a}, \mathrm{~N}(\%)$ & Morning $^{b}, \mathrm{~N}(\%)$ \\
\hline MTBC & $117(1.6)$ & $149(1.9)$ & $47(0.6)$ & $53(0.7)$ \\
NTM & $294(4.1)$ & $312(4.1)$ & $80(1.1)$ & $87(1.1)$ \\
Negative & $5878(80.7)$ & $5944(76.7)$ & $6868(94.2)$ & $6966(90.2)$ \\
Contaminated & \\
Totally Contaminated & $993(13.6)$ & $1307(16.9)$ & $424(5.7)$ & $654(8.4)$ \\
\hline
\end{tabular}

${ }^{a}$ Specimen taken on the spot; ${ }^{b}$ Specimen taken early morning; No growth after eight weeks of incubation; ${ }^{d}$ Tubes that have either spot only or morning only contaminated; ${ }^{\top}$ Tubes that have both spot and morning contaminated; LJ: Lowenstein-Jensen; MGIT: Mycobacterial Growth Indicator Tube; MTBC: Mycobacterium tuberculosis complex; NTM: Non-tuberculous mycobacteria. 


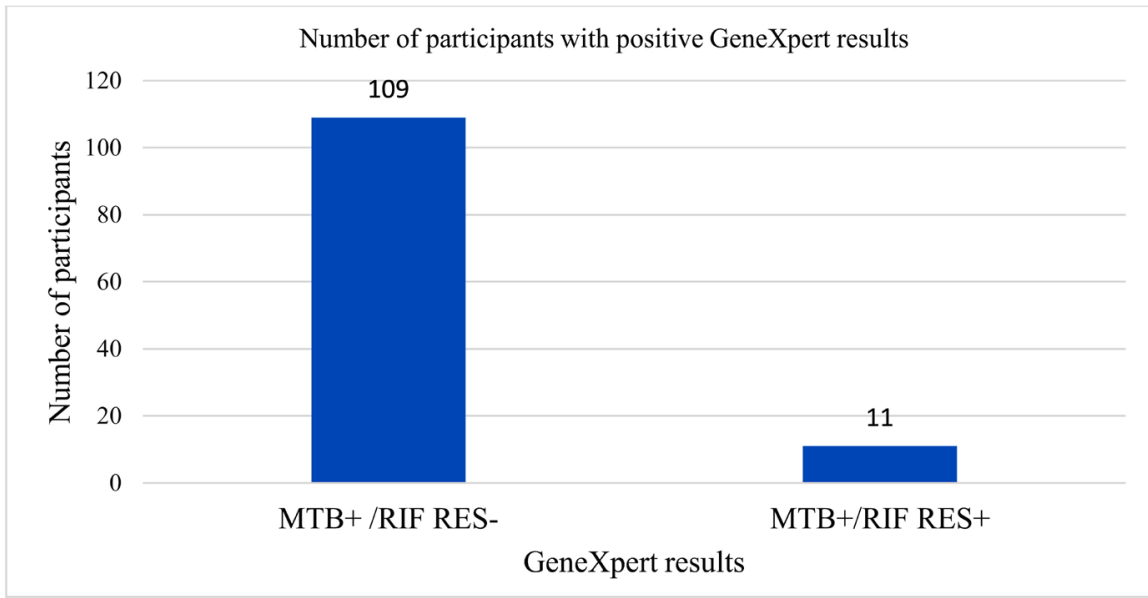

MTB+/RIF RES- = M. tuberculosis detected; Rifampicin resistance not detected. MTB+/RIF RES+ = M. tuberculosis detected; Rifampicin resistance detected.

Figure 2. Patterns of GeneXpert results.

were run on GeneXpert. Mycobacterium tuberculosis was detected in at least one sputum specimen of 109 participants. Eleven out of the 109 participants had their specimens being rifampicin resistant (Figure 2). Prevalence of smear positive TB and bacteriologically confirmed TB among adult population ( $\geq 15$ years) was estimated at $111(95 \%$ CI: 76,145) and 356 (95\% CI: 288,425) per 100,000 population respectively.

\section{Discussion and Lessons Learnt}

The overall goal of the prevalence survey was to gain better understanding of the burden of TB disease and identify ways by which TB control can be improved. The laboratory played a very important role in the successful conduct and outcome of the survey. Sample collection and processing were all performed by the laboratory which generated data for calculating the prevalence of TB disease. The proportion of survey participants eligible for sputum collection was higher (13.4\%) than what was reported in other African countries such as Ethiopia (13\%) [5] and Nigeria (10.6\%) [6], but lower than in Zambia (14.6\%) [7] and Gambia (13.8\%) [8]. The high sputum collection rate (98\% for at least one specimen and $93 \%$ for both spot and morning specimen) was comparable to results from surveys in other countries [5] [7] [8]. In the case of Ghana, we can attribute this finding to factors such as effective community sensitization prior to commencement of the survey, professional skill of the survey team, supervision by field laboratory staff whereby in some instances, follow-up to residences of participants for specimen collection and the provision of digital chest X-ray examination. In addition, those who returned the early morning sputum sample were also given a vitamin fortified cereal.

Smear results from majority of the specimens were negative which was much expected in a TB prevalence survey where in addition to the gold standard muco-purulent sputum, salivary specimens are accepted [9]. It was also observed 
that smear positivity were of the lower grade (scanty) and this could be due to the paucibacillary load of specimens received. Thus, the quality and bacillary load of sputum specimens collected as well as method used can determine AFB detection rate [10]. The high number of smear negative-culture positive specimens from this survey confirms the general trends observed in surveys from other countries [5] [6] [7] [8]. We could safely assumed that these culture positive cases would have been missed if microscopy was the only tool used for detecting TB in the survey. Both spot and morning specimens for MGIT and LJ culture as used in this survey were similar to what was used in Zambia, Cambodia and Lao [8] [11] [12], although both specimens were used for only one culture method in these instances. On the contrary, the surveys in Pakistan [13] and Vietnam [14] used one specimen one culture method. MGIT culture positivity (isolation of both MTBC and NTM) was higher than LJ. This result is expected results due to the high recovery rate and short detection time of the MGIT system and this was consistent with reports from other studies [15] [16] [17]. Due to the extremely low mycobacterial growth on the LJ medium, only MGIT culture positive as well as ZN stained positive results are considered in the definition of a case of TB and hence in the calculation of the TB prevalence rate in this study. The relatively high recovery rate of NTM underscores the need for culture and subsequent identification of mycobacterial species since microscopy alone cannot distinguish between MTBC and NTM. A significant feature of this survey which was also used during the Zambia survey was the use of GeneXpert to confirm all smear positive cases and contaminated culture provided their decontaminated sediments were available. For instance, Mycobacterium tuberculosis was detected in $14(26.9 \%)$ out of 52 available sediments whose cultures were totally contaminated. Of the 14 GeneXpert positive sediments, one was rifampicin resistant. These cases among others would have gone away undetected and might pose a threat to public health. High contamination rates (20\%-30\%) were observed during the initial stages of the survey period due to some operational challenges. The most common types of contamination were fungal and non-mycobacterial growth leading to liquefying or discolouration of inoculated LJ media and homogenous turbidity of MGIT culture. As expected, contamination rate of the MGIT were higher than LJ cultures. Also, majority of the contaminated tubes of both media were those inoculated with the morning specimen. This may be due to the fact that the participants were not directly supervised by the field staff during the production of the morning specimens which could result in contamination with food debris in the mouth unlike the spot one whereby cleaned water was used to rinse the mouth of participants before sputum production. However, due to mitigating measures put in place by laboratory staff with support from the survey coordinating team, the contamination rates declined steadily (13\% - 17\% for MGIT and 5\% - 9\% for LJ) till the end of the survey period. These rates were still higher than the general recommendation of $5 \%$ for all media types although up to $7 \%$ - $8 \%$ may be accepted for liquid media [18]. 


\section{Strengths and Limitations}

We highlight here some of the strengths of the laboratory that contributed to the success of the survey. Firstly, the use of two specimens (both spot and morning) as well as two methods for both microscopy and culture accounted for the availability of over $90 \%$ of results. Next, specimens were readily processed anytime they arrived from the field due to a 24-hour shift system the laboratory staff run. Thus, except in very rare cases there were little delays for inoculation from time of sputum collection (i.e. within 5 days). Furthermore, the use of fully electronic data collection and entry barcode systems ensured minimal human error. By this, some health facilities have shifted from the fully manual system of data collection and management to semi-automated systems. Generally, through the survey, the capacity to conduct laboratory examinations such as microscopy (ZN/AO), culture (MGIT/LJ) and GeneXpert have been enhanced. Despite these strengths, some challenges were also encountered. Key among these were the relatively high contamination rates of specimens in the initial stages of the survey which could have underestimated the TB prevalence. Furthermore, due to the high numbers and frequency of specimens received in the laboratory, there could be some possible cases of cross-contamination. However, during the analysis these challenges were treated by imputation analysis. Another notable challenge was the sudden breakdown of the biosafety cabinet in which the samples were being processed at one of the two laboratories. This necessitated temporary suspension of laboratory work for maintenance, and thus delayed inoculation of some of the collected specimens around that period.

\section{Implication for TB Control by NTP}

The experiences gained by the laboratory staff have contributed immensely to the improvement of some activities of the NTP. Among these are installation of GeneXpert and hands on training at some regional and district hospitals by some laboratory staff who were involved in the prevalence survey.

\section{Conclusion and Recommendation}

In this study, most of the specimens were smear negative but culture positive. Also, significant number of smear positive specimens was found to be NTM which indicates that relying on smear microscopy alone may lead to misdiagnosis and unnecessary treatment leading to development of drug resistance. Based on these findings and from the laboratory staff point of view we recommend that a wide roll out of novel technologies such as GeneXpert be implemented nationwide. This may increase case detection and minimize false-positive smear and false-negative culture results. Finally, the capacity and skills of the laboratory staff must be enhanced through regular in-service training and other refresher courses. 


\section{Acknowledgements}

The authors are grateful to all study participants as well as staff involved in the TB prevalence survey.

\section{Competing Interest}

The authors declare that they have no financial or personal relationship(s) that may have inappropriately influenced them in writing this article.

\section{Authors' Contributions}

KKA and FAB came out with the study design. KKA supervised the laboratory work and contributed to the writing and editing of the manuscript. SOA, CB, EM, SE, PD, MAO, SK, HG, SKA performed the laboratory work. SOA contributed to the manuscript writing and data analysis. FAB contributed to the study implementation by providing reagents and equipment. All authors read and approved the final manuscript before submission.

\section{Funding}

We are grateful to the Global Fund against HIV/AIDS, TB \& Malaria, WHO, Dutch Government and the Ghana Health Service for their financial support. The publication of the study was funded by DELTAS Africa Initiative [Afrique One-ASPIRE/DEL-15-008]. Afrique One-ASPIRE is funded by a consortium of donors including the African Academy of Sciences (AAS), Alliance for Accelerating Excellence in Science in Africa (AESA), the New Partnership for Africa's Development Planning and Coordinating (NEPAD) Agency, the Wellcome Trust $[107753 / \mathrm{A} / 15 / \mathrm{Z}]$ and the UK government.

\section{Disclaimer}

The views expressed in the submitted article are that of the authors and not an official position of their respective institution or funders.

\section{References}

[1] World Health Organization (2014) Global Tuberculosis Report. https://www.who.int/tb/publications/global_report/gtbr14_executive_summary.pdf

[2] World Health Organization (2015) Global Tuberculosis Report, 2015. http://apps.who.int/iris/bitstream/10665/191102/1/9789241565059_eng.pdf

[3] World Health Organization (2013) Global Tuberculosis Report, 2013. http://apps.who.int/iris/bitstream/10665/91355/1/9789241564656_eng.pdf

[4] World Health Organization (2010) Tuberculosis Prevalence Surveys: A Handbook. 2nd Edition, Geneva, 324.

[5] Kebede, A.H., Alebachew, Z., Tsegaye, F., Lemma, E., Abebe, A., Agonafir, M., et al. (2014) The First Population-Based National Tuberculosis Prevalence Survey in Ethiopia, 2010-2011. International Journal of Tuberculosis and Lung Disease, 18, 635-639. https://doi.org/10.5588/ijtld.13.0417

[6] Federal Republic of Nigeria (2014) Report: First National Tuberculosis Prevalence 
Survey 2012, Nigeria. Abuja.

[7] Kapata, N., Chanda-Kapata, P., Ngosa, W., Metitiri, M., Klinkenberg, E., Kalisvaart, N., et al. (2016) The Prevalence of Tuberculosis in Zambia: Results from the First National Tuberculosis Prevalence Survey, 2013-2014. PLoS ONE, 11, e0146392. https://doi.org/10.1371/journal.pone.0146392

[8] Adetifa, I.M.O., Kendall, L., Bashorun, A., Linda, C., Omoleke, S., Jeffries, D., et al. (2016) A Tuberculosis Nationwide Prevalence Survey in Gambia, 2012. Bulletin of the World Health Organization, 94, 433-441.

https://doi.org/10.2471/BLT.14.151670

[9] Kim, S.J. (2017) Laboratory Works in TB Prevalence Survey. http://www.who.int/tb/advisory_bodies/impact_measurement_taskforce/meetings/ prevalence_survey/psws_lab_works_kim.pdf?ua $=1$

[10] Abraham, P.R., Sharma, V.D. and Shivannavar, C.T. (2012) Diagnosis of TB from Smear \& Culture Negative Sputum Specimens by IS 6110 Based PCR. Indian Journal of Medical Research, 135, 249-251.

[11] Eang, M.T., Okada, K., Yamada, N., Satha, P., Ota, M., Saint, S., et al. (2014) Cross-Sectional Studies of Tuberculosis Prevalence in Cambodia between 2002 and 2011. Bulletin of the World Health Organization, 92, 573-581. https://doi.org/10.2471/BLT.13.131581

[12] Law, I., Sylavanh, P., Bounmala, S., Nzabintwali, F., Paboriboune, P., Iem, V., et al. (2015) The First National Tuberculosis Prevalence Survey of LFM PDR (20102011). Tropical Medicine \& International Health, 20, 1146-1154. https://doi.org/10.1111/tmi.12536

[13] Qadeer, E., Fatima, R., Yaqoob, A., Tahseen, S., Ul Haq, M., Ghafoor, A., et al. (2016) Population Based National Tuberculosis Prevalence Survey among Adults (> 15 Years) in Pakistan, 2010-2011. PLoS ONE, 11, e0148293. https://doi.org/10.1371/journal.pone.0148293

[14] Hoa, N.B., Sy, D.N., Nhung, N.V., Tiemersma, E.W., Borgdorff, M.W. and Cobelens, F.G. (2010) National Survey of Tuberculosis Prevalence in Vietnam. Bulletin of the World Health Organization, 88, 273-280. https://doi.org/10.2471/BLT.09.067801

[15] Chihota, V.N., Grant, A.D., Fielding, K., Ndibongo, B., van Zyl, A., Muirhead, D. and Churchyard, G.J. (2010) Liquid vs. Solid Culture for Tuberculosis: Performance and Cost in a Resource Constrained Setting. International Journal of Tuberculosis and Lung Disease, 14, 1024-1031.

[16] Lee, J.J., Suo, J., Lin, C.B., Wang, J.D., Lin, T.Y. and Tsai, Y.C. (2003) Comparative Evaluation of the Bactec MGIT 960 System with Solid Medium for Isolation of Mycobacteria. International Journal of Tuberculosis and Lung Disease, 7, 569-574.

[17] Muyoyeta, M., Schaap, J.A., De Haas, P., et al. (2009) Comparison of Four Culture Systems for Mycobacterium tuberculosis in the Zambian National Reference Laboratory. International Journal of Tuberculosis and Lung Disease, 13, 460-465.

[18] Kent, P.T. and Kubica, G.P. (1985) Public Health Microbiology, a Guide for the Level III Laboratory. Centers for Disease Control, Division of Laboratory Training and Consultation, US Department of Health and Human Services, US Government Printing Office, Atlanta. 


\section{List of Abbreviations}

AFB: Acid Fast Bacilli

AO: Auramine O

BSL3: Biosafety Level 3

FM: Fluorescence Microscopy

GeneXpert: Xpert ${ }^{\mathbb{B}}$ MTB/RIF assay

HPF: High Power Field

ID: Identification

KBTH: Korle-Bu Teaching Hospital

KCCR: Kumasi Centre for Collaborative Research

LJ: Lowenstein-Jensen

MGIT: Mycobacterial Growth Indicator Tube

MTBC: Mycobacterium tuberculosis complex

$\mathrm{NaOH}-\mathrm{NALC}$ : Sodium hydroxide-N-acetyl L-cysteine

NMIMR: Noguchi Memorial Institute for Medical Research

NTP: National Tuberculosis Programme

NTM: Non-tuberculous mycobacteria

PBS: Phosphate buffer saline

WHO: World Health Organization

WHO/IUATLD: World Health organization/International Union against Tuberculosis and Lung Diseases

WHO/NICD: World Health Organization/National Institute for Communicable Diseases

ZN: Ziehl-Nielsen 